\title{
Outbreak of Infections by Hepatitis B Virus Genotype A and Transmission of Genetic Drug Resistance in Patients Coinfected with HIV-1 in Japan ${ }^{\nabla}$
}

\author{
Seiichiro Fujisaki, ${ }^{1}$ Yoshiyuki Yokomaku, ${ }^{1}$ Teiichiro Shiino, ${ }^{2}$ Tomohiko Koibuchi, ${ }^{3}$ Junko Hattori, ${ }^{1}$ \\ Shiro Ibe, ${ }^{1}$ Yasumasa Iwatani, ${ }^{1,4}$ Aikichi Iwamoto, ${ }^{3}$ Takuma Shirasaka, ${ }^{5}$ \\ Motohiro Hamaguchi, ${ }^{6}$ and Wataru Sugiura ${ }^{1,4 *}$ \\ Department of Infectious Diseases and Immunology, Clinical Research Center, National Hospital Organization, Nagoya Medical Center, \\ Nagoya, Japan ${ }^{1}$; Infectious Disease Surveillance Center, National Institute of Infectious Diseases, Tokyo, Japan ${ }^{2}$; \\ Institute of Medical Science, The University of Tokyo, Tokyo, Japan ${ }^{3}$; Department of AIDS Research, \\ Nagoya University Graduate School of Medicine, Nagoya, Japan ${ }^{4}$; AIDS Medical Center, \\ National Hospital Organization, Osaka National Hospital, Osaka, Japan ${ }^{5}$; and \\ Aichi Blood Center, Japanese Red Cross Society, Nagoya, Japan ${ }^{6}$
}

Received 24 October 2010/Returned for modification 2 December 2010/Accepted 8 January 2011

\begin{abstract}
The major routes of hepatitis B virus (HBV) infection in Japan has been mother-to-child transmission (MTCT) and blood transfusion. However, HBV cases transmitted through sexual contact are increasing, especially among HIV-1-seropositive patients. To understand the molecular epidemiology of HBV in HBV/ HIV-1 coinfection, we analyzed HBV genotypes and HIV-1 subtypes in HBV/HIV-1-coinfected patients at Nagoya Medical Center from 2003 to 2007. Among 394 HIV-1-infected Japanese men having sex with men (MSM) who were newly diagnosed during the study period, $31(7.9 \%)$ tested positive for the hepatitis B virus surface antigen. HBV sequence analyses were successful in 26 cases, with $21(80.7 \%)$ and $5(19.3 \%)$ cases determined as genotypes $\mathrm{A}$ and $\mathrm{C}$, respectively. Our finding that HBV genotype $\mathrm{A}$ was dominant in HIV-1seropositive patients alerts clinicians to an alternative outbreak of HBV genotype A in the HIV-1-infected MSM population and a shift in HBV genotype from $\mathrm{C}$ to $\mathrm{A}$ in Japan. The narrow genetic diversity in genotype A cases suggests that genotype A has been recently introduced into the MSM population and that sexual contacts among MSM were more active than speculated from HIV-1 tree analyses. In addition, we found a lamivudine resistance mutation in one naïve case, suggesting a risk of drug-resistant HBV transmission. As genotype A infection has a higher risk than infection with other genotypes for individuals to become HBV carriers, prevention programs are urgently needed for the target population.
\end{abstract}

The number of hepatitis B virus (HBV)-infected persons in Japan is estimated to be 1 million, or $0.8 \%$ of the total population (31). HBV is classified into eight genotypes, A to $\mathrm{H}$, by their differences in genome sequences $(11,12,22)$. Circulating genotypes in Japan differ according to geographical region, with the prevalent genotypes in 2001 being $\mathrm{C}(84.7 \%)$ and $\mathrm{B}$ $(12.2 \%)$, while $\mathrm{A}(1.7 \%)$ and $\mathrm{D}(0.4 \%)$ were less frequent (17). HBV infection in Japan has been transmitted mainly by two routes, mother-to-child transmission (MTCT) and blood transfusion, which have been targeted by prevention programs still being operated today $(13,15-17,25)$.

Regarding MTCT, all pregnant women are screened for HBV antigen and antibody. Mothers who are HBV infected are prohibited from breast-feeding, and their newborns are vaccinated against HBV. Regarding infection by blood transfusion, all donated blood is tested by anti-hepatitis B surface antibody (HBsAb) testing and PCR to exclude HBV-contaminated blood from the supply. These prevention programs have

\footnotetext{
* Corresponding author. Mailing address: Department of Infection and Immunology, Clinical Research Center, Nagoya Medical Center, 4-1-1 Sannomaru, Nakaku, Nagoya 4600001, Japan. Phone: 81-52-9511111. Fax: 81-52-963-3970. E-mail: wsugiura@nnh.hosp.go.jp.

${ }^{\nabla}$ Published ahead of print on 19 January 2011.
}

been successful, and the risks of HBV infection by these two routes have been reduced dramatically.

However, HBV infection by sexual contact has recently become a prevailing alternative transmission route of $\mathrm{HBV}$ in Japan $(30,36)$. In particular, coinfection with HBV and human immunodeficiency virus type 1 (HIV-1), the causative agent of AIDS, has been increasing among men who have sex with men (MSM), and the incidence of HBV infection associated with HIV-1-seropositive cases appeared to be $8.8 \%$, which is higher than that in the general population (5). Thus, the epidemiology of HBV infection in Japan is quickly shifting. Here we report the most recent molecular epidemiologic status of HBV/HIV-1 coinfection.

\section{MATERIALS AND METHODS}

Sample. HIV/AIDS patients newly diagnosed at Nagoya Medical Center from 2003 to 2007 were tested for hepatitis B surface antigen (HBsAg), and HBsAgpositive patients were enrolled in the study. Clinical data (age, gender, suspected route of HIV-1 infection, aspartate aminotransferase [AST] and alanine aminotransferase [ALT] plasma levels, CD4-positive T cell count, and HIV viral load) were obtained from medical records. Plasma HBV viral load was measured with COBAS TaqMan (Roche Diagnostics, Basel, Switzerland), and plasma HBc IgM titer was measured with Lumipulse (Fujirebio, Tokyo, Japan). The time of HBV infection was estimated by patient interview and HBc IgM titer results. This study was conducted according to the principles expressed in the Declaration of Helsinki. The study was approved by the Institutional Review Boards of the National Institute of Infectious Diseases and Nagoya Medical Center. All pa- 


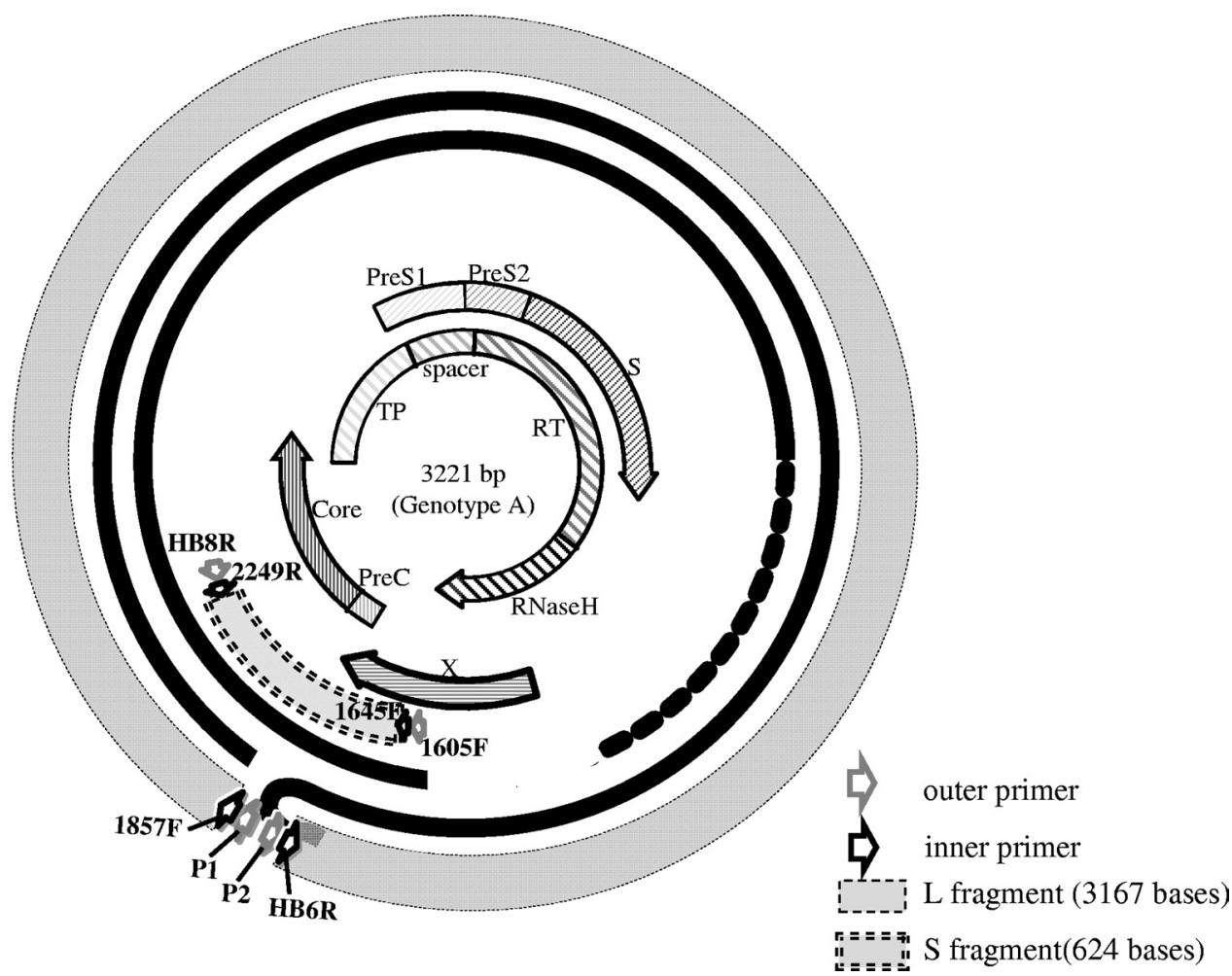

FIG. 1. Genetic regions of HBV and HIV-1 used for phylogenetic tree analyses. The whole HBV genome was amplified in two fragments, L and $\mathrm{S}$, and assembled. $\mathrm{L}$ and $\mathrm{S}$ fragments are indicated by single and double dashed lines, respectively.

tients provided written informed consent for collection of samples and subsequent analysis.

Amplification of HBV and HIV DNA fragments and determination of DNA sequences. HBV nucleic acid was extracted from plasma using a MagNA Pure Compact Nucleic Acid Isolation Kit I (Roche Diagnostics). As shown in Fig. 1, the full-length HBV genome was amplified in two fragments, $\mathrm{L}$ (3,167 bases) and S (624 bases). The primers used for amplifying HBV DNA were both newly designed and have been published previously (27). Details of these primers are summarized in Table 1. The DNA polymerases used for the first and nested
PCRs were LA Taq (Takara, Shiga, Japan) and Prime Star HS (Takara) polymerase, respectively. The HBV genotypes were also determined using a commercial kit (Institute of Immunology, Tokyo, Japan) based on enzyme immunoassay to confirm that the results did not differ from those based on phylogenetic tree analysis.

The HIV-1 gag p17 (396 bp [bp 790 to 1185]), pol (1,117 bp [bp 2253 to 3369]), and env C2V3 (222 bp [bp 6996 to 7217]) regions were amplified from extracted plasma HIV-1 RNA by reverse transcription-PCR (RT-PCR) using the SuperScript one-step RT-PCR system for long templates (Invitrogen, Carlsbad, CA)

TABLE 1. Primers for amplifying the HBV and HIV-1 genomes

\begin{tabular}{|c|c|c|c|}
\hline Name & Direction $^{a}$ & Sequence $\left(5^{\prime} \rightarrow 3^{\prime}\right)$ & Region \\
\hline P1 & $\mathrm{F}$ & ТTTТСАССТСТGССТААТСА & First PCR, HBV L fragment \\
\hline $\mathrm{P} 2$ & $\mathrm{R}$ & AAAAAGTTGCATGGTGCTGG & First PCR, HBV L fragment \\
\hline $1605 \mathrm{~F}$ & $\mathrm{~F}$ & CGCATGGAGACCACCGTGAA & First PCR, HBV S fragment \\
\hline HB8R & $\mathrm{R}$ & ATAGGGGCATTTGGTGGTCT & First PCR, HBV S fragment \\
\hline $1857 \mathrm{~F}$ & $\mathrm{~F}$ & CTACTGTTCAAGCCTCCAAG & Nested PCR, HBV L fragment \\
\hline HB6R & $\mathrm{R}$ & AACAGACCAATTTATGCCTA & Nested PCR, HBV L fragment \\
\hline $1645 \mathrm{~F}$ & $\mathrm{R}$ & AGGTCTTGCATAAGAGGACT & Nested PCR, HBV S fragment \\
\hline 2249R & $\mathrm{F}$ & CСAAAAGACACCAAATAYTC & Nested PCR, HBV S fragment \\
\hline $172 \mathrm{~A}$ & $\mathrm{~F}$ & ATCTCTAGCAGTGGCGCCCGAACAG & RT-PCR, HIV-1 gag fragment \\
\hline 173B & $\mathrm{R}$ & CTGATAATGCTGAAAACATGGGTAT & RT-PCR, HIV-1 gag fragment \\
\hline 174A & $\mathrm{F}$ & CTCTCGACGCAGGACTCGGCTTGCT & Nested PCR, HIV-1 gag fragment \\
\hline 175B & $\mathrm{R}$ & CCCATGCATTCAAAGTTCTAGGTGA & Nested PCR, HIV-1 gag fragment \\
\hline K1 & $\mathrm{F}$ & AAGGGCTGTTGGAAATGTGG & RT-PCR, HIV-1 pol fragment \\
\hline U13 & $\mathrm{R}$ & CCCACTCAGGAATCCAGGT & RT-PCR, HIV-1 pol fragment \\
\hline K4 & $\mathrm{F}$ & GAAAGGAAGGACACCAAATGA & nested PCR, HIV-1 pol fragment \\
\hline U12 & $\mathrm{R}$ & CTCATTCTTGCATATTTTCCTGTT & Nested PCR, HIV-1 pol fragment \\
\hline 106A & $\mathrm{F}$ & CATACATTATTGTGCCCCGGCTGG & RT-PCR, HIV-1 env fragment \\
\hline 17B & $\mathrm{R}$ & AGAAAAАТTССССТСТАСААТTАА & RT-PCR, HIV-1 env fragment \\
\hline $14 \mathrm{~A}$ & $\mathrm{~F}$ & AATGTCAGCTCAGTACAATGCACAC & Nested PCR, HIV-1 env fragment \\
\hline 10B & $\mathrm{R}$ & ATTTCTGGGTCCCCTCCTGAGG & Nested PCR, HIV-1 env fragment \\
\hline
\end{tabular}

\footnotetext{
${ }^{a} \mathrm{~F}$, forward; R, reverse.
} 
TABLE 2. HBV genotype reference sequences collected from the DNA Database of Japan (DDBJ) for tMRCA analysis

\begin{tabular}{|c|c|}
\hline Genotype & DDBJ accession no. \\
\hline \multicolumn{2}{|c|}{$\begin{array}{c}\text { A } \\
\text { GQ486599, EU414132 }\end{array}$} \\
\hline B........ & ...FJ751547, GQ924611 \\
\hline C....... & ...GQ924615, GQ486096, EU939589, GQ486684 \\
\hline D........ & $\begin{array}{l}\text { GQ486337, FJ349228, GQ924652, EU414124, } \\
\text { GQ922001, GQ486586 }\end{array}$ \\
\hline & ...GQ486756, GQ161830, FJ349237 \\
\hline F ........ & ...GQ486537, GQ486515, GQ486570 \\
\hline G......... & ...GQ486843 \\
\hline $\mathrm{H} \ldots \ldots$. & ...GQ486592, AB266536 \\
\hline
\end{tabular}

followed by a second PCR using LA Taq polymerase. The primers used for HIV-1 sequencing are also summarized in Table 1 . The amplicons were purified using a MultiScreen PCR filter plate (Millipore, Billerica, MA), and the sequencing reaction was performed using the BigDye Terminator v3.1 cycle sequencing kit (Applied Biosystems, Carlsbad, CA) and analyzed with the ABI PRISM 3130 (Applied Biosystems) autosequencer. Electropherograms were edited and verified by SeqScape v2.5 software (Applied Biosystems).

Phylogenetic tree analyses and genotype determination. HBV genotypes were determined by phylogenetic tree analysis with reference sequences. HBV sequences were aligned with 23 reference sequences from the National Center for Biotechnology Information (NCBI) database by using the CLUSTAL W program and analyzed by Kimura two-parameter methods. Genetic distances were calculated by the maximum composite likelihood, and phylogenetic trees were constructed by the neighbor-joining method using MEGA version 4 software. The reliabilities of branches were evaluated by bootstrap analysis with 1,000 replicates.

Phylogenetic trees of the HIV-1 gag, pol, and env regions were also constructed with 62 HIV-1 reference sequences obtained from the HIV-1 sequence database (Los Alamos National Laboratory).

Estimated tMRCAs. Evolutionary rates, chronological phylogenies, and other evolutionary parameters of HBV genotypes were estimated from heterochronous data for the HBV genomic sequences collected in our study, together with reference sequences from public databases (Table 2), using the Bayesian Markov chain Monte Carlo (MCMC) method. The nucleotide substitution model was evaluated by the hierarchical likelihood ratio test using PAUP v4.0 (29) with MrModeltest (14) and the general time-reversible (GTR) model with both invariant site (I) and gamma-distributed site (G) heterogeneity for four rate categories showing maximum likelihood. Bayesian MCMC analyses were performed with BEAST v1.4.8 (4) using the substitution model of GTR + I + G, three partitions into codon positions, and a relaxed molecular clock model (the uncorrelated log normal-distributed model) (3). Four different population dynamic models (exponential growth, logistic growth, constant population, and Bayesian skyline plot [BSP]) were tested in the analyses. According to BSP properties, constant-growth models were adopted for the HBV genome sequences. Each Bayesian MCMC analysis was run for 40 million states and sampled every 10,000 states. Posterior probabilities were calculated with a burn-in of 4 million states and checked for convergence using Tracer v1.4 (21). The maximum clade credibility tree for analyzing the MCMC data set was annotated by TreeAnnotator in the BEAST package. The posterior distribution of the substitution rate obtained from the heterochronous sequences was subsequently incorporated as a prior distribution for the mean evolutionary rate of the HBV genome, thereby adding a time scale to the phylogenetic histories of the given viruses and enabling estimation of the time of the most recent common ancestor (tMRCA) (19).

Determination of $\mathrm{HBV}$ drug resistance mutations. HBV cases resistant to nucleoside analogue reverse transcriptase inhibitors (NRTI) were determined by analyzing amino acid sequences of the RT region. The approved anti-HBV drugs in Japan are lamivudine, adefovir, and entecavir. In cases of HBV/HIV-1 coinfection, tenofovir and emtricitabine are also used. We studied whether the viruses have drug resistance mutations against these antiretroviral drugs with or without a history of antiretroviral treatments and confirmed the following resistance mutations: lamivudine/emtricitabine resistance mutations V173L, L180M, and M204I/V; adefovir resistance mutations A181V, I233V, and N236T; entecavir resistance mutations I169T, L180M, T184G, S202I, M204I/V, and M250V; and tenofovir resistance mutation A194T (1, 2, 24, 32, 34, 35). Furthermore, major drug resistance mutations in HIV-1 were defined according to the criteria of the International AIDS Society (IAS)-USA and Stanford HIV drug resistance database $(7,23)$

\section{RESULTS}

The major HBV genotype circulating among Japanese MSM is genotype A. During the study period, 394 cases were newly diagnosed as HIV/AIDS, and 31 cases were determined as HBsAg positive. Thus, the average prevalence of HBV/HIV-1 coinfection in our study population was $7.9 \%$. Analysis of the coinfection prevalence in each year showed increases from 2.8 to $3.3 \%$ in 2003 to 2004 and from 7.4 to $13.2 \%$ in 2005 to 2007 (Fig. 2). As the suspected route of HIV-1 infections in all 31 cases was MSM, HBV appears to be quickly spreading among the MSM population. Of these HBV/HIV-1-coinfected cases, 26 isolates were successfully sequenced for both HBV and HIV-1, and their subtypes and genotypes were determined. Regarding the five cases for which the HBV genome could not be sequenced, plasma HBV DNA copies were undetectable in four cases, and low $\left(10^{3.3}\right.$ copies $\left./ \mathrm{ml}\right)$ in one case.

The median age of the patients was 34 years (interquartile range [IQR], 29.5 to 37.0 ) (Table 3). The median plasma viral loads of HBV and HIV-1 were $4.4 \times 10^{8}\left(\mathrm{IQR}, 4.9 \times 10^{4}-\right.$ $\left.6.3 \times 10^{8}\right)$ and $6.4 \times 10^{4}\left(\mathrm{IQR}, 2.0 \times 10^{4-2} .0 \times 10^{5}\right) \mathrm{copies} / \mathrm{ml}$, respectively. Hepatitis $\mathrm{B}$ core antigen $(\mathrm{HBcAg}) \mathrm{IgM}$ was positive in nine patients, of which two were suspected to harbor acute HBV infection according to their HBsAg positivity, AST and ALT plasma levels, and patient interviews. The other 7 $\mathrm{HBcAg}$-positive patients were categorized as having acute hepatitis or exacerbated chronic hepatitis, and $17 \mathrm{HBcAg}$-negative patients were determined as being in the chronic hepatitis stage.

According to phylogenetic tree analysis, 26 cases were classified into two genotypes, either A or C. As shown in Fig. 3, 21 and 5 cases were classified as genotypes $\mathrm{A}$ and $\mathrm{C}$, respectively. The subgenotypes of the 21 genotype A cases were all A2, the predominant subgenotype in Europe and North America, whereas the subgenotypes of the 5 genotype $\mathrm{C}$ cases were all $\mathrm{C} 1$, the most prevalent subgenotype in eastern Asia, including Japan, South Korea, and northern China. Genotype B, the

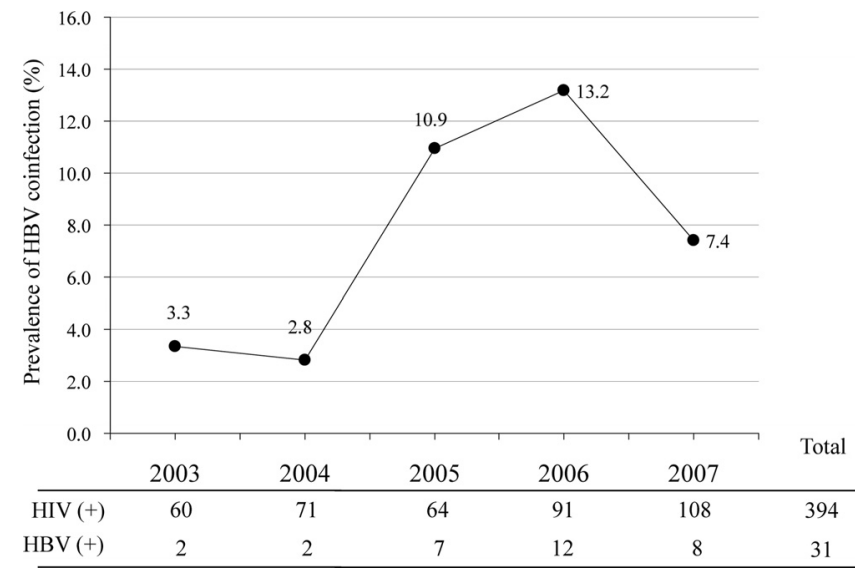

FIG. 2. Transitions in HBV infection rates in HBV/HIV-1-coinfected patients. HBV infection rates are plotted versus year, with the numbers of HIV-1-infected and HBV/HIV-1-coinfected patients shown below the $x$ axis. 


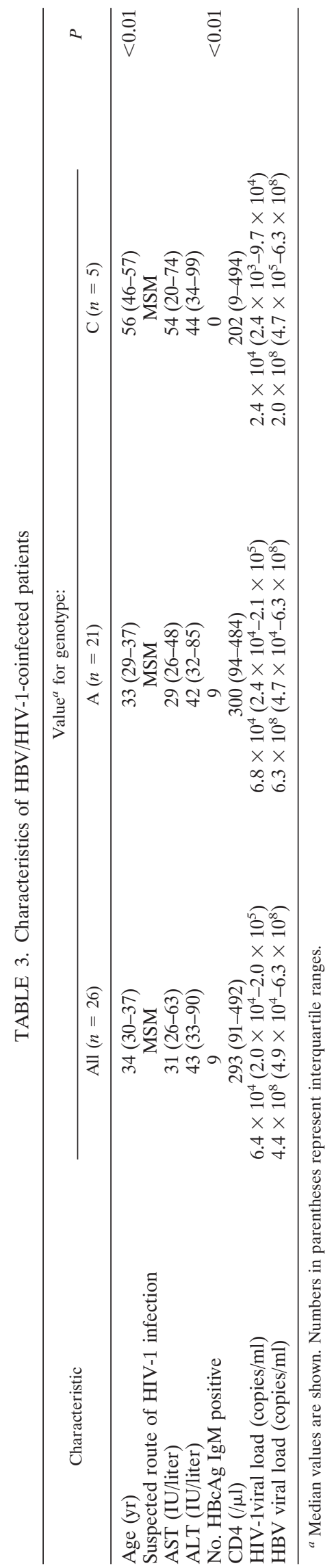

second most predominant HBV genotype in Japan, was not detected in our study. Interestingly, the genotype A and C populations showed obvious differences in genetic diversity. The 21 group A2 samples (Fig. 3) formed a cluster with little

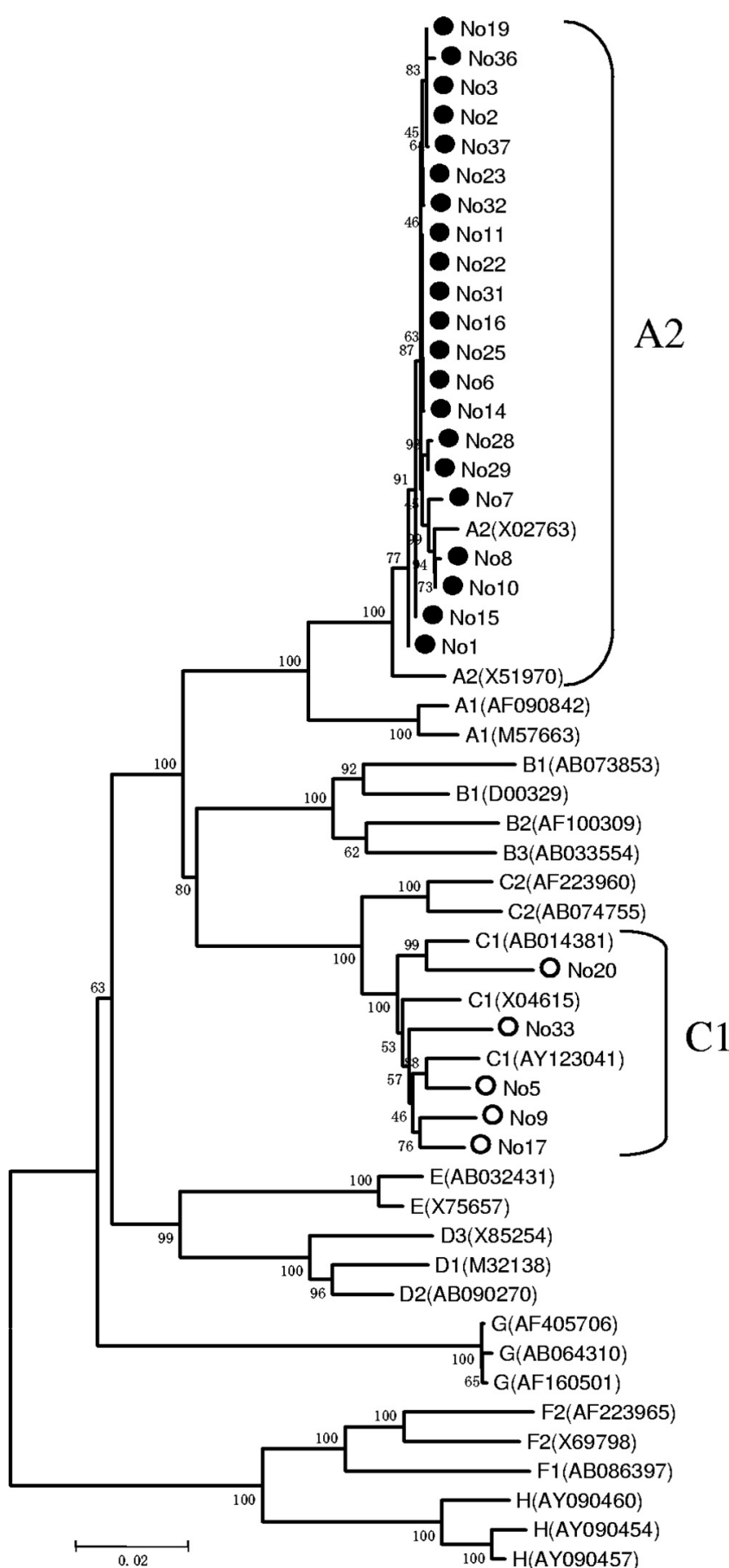

FIG. 3. Phylogenetic tree analyses of HBV isolated from HBV HIV-1-coinfected patients. The phylogenetic tree was constructed using 26 full-length HBV genome sequences detected in HBV/HIV-1coinfected patients in Nagoya (both solid and open circles) and 23 reference sequences from the NCBI database. Twenty-one and five cases were distributed in the clusters of genotype A (solid circles) and $\mathrm{C}$ (open circles), respectively. 

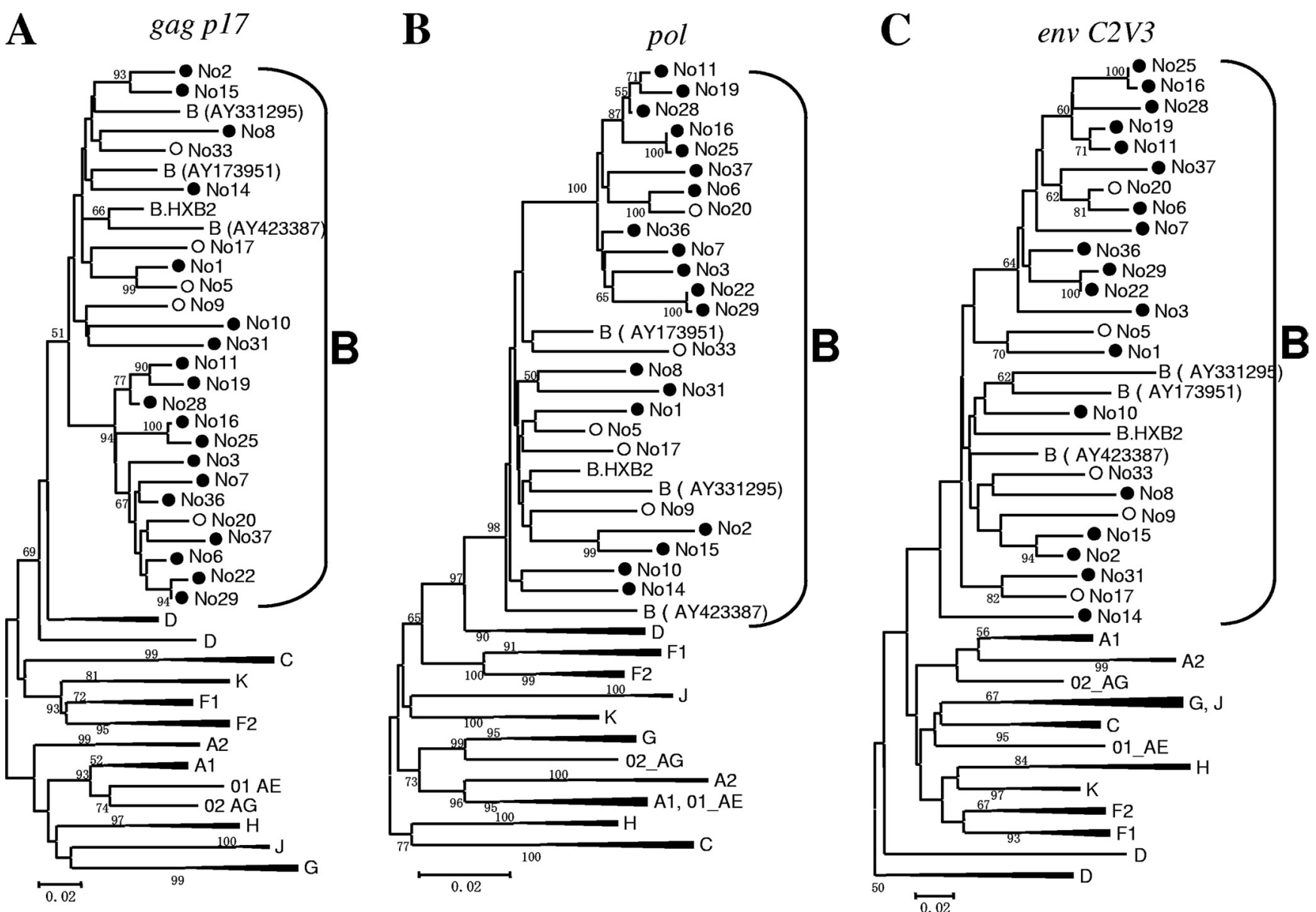

FIG. 4. Phylogenetic tree analyses of HIV-1 isolated from HBV/HIV-1-coinfected patients. Phylogenetic trees were constructed using the 25 HIV-1 sequences obtained in this study and 62 HIV-1 reference sequences from the Los Alamos National Laboratory database. The nucleotide base sequences of gag p17 (A), pol PR to RT (B), and env C2V3 (C) gene regions were analyzed. In all analyses, all the HIV-1-positive cases detected in Nagoya (both solid and open circles) were distributed in the subtype B cluster. Cases of coinfection with genotype C HBV are shown with open circles.

or no genetic distance between each other, indicating their extremely close genetic relationships. In contrast, the five group $\mathrm{C} 1$ cases did not form a single cluster and had longer branches than those of group A2.

Patients with genotypes $\mathrm{A}$ and $\mathrm{C}$ also differed significantly in age (Table 3 ). The median age of the genotype A patients was 33 years (IQR, 29 to 37), whereas that of the genotype C patients was 56 (IQR, 46 to 57$)(P<0.01)$. Furthermore, all nine $\mathrm{HBcAg}$ IgM-positive cases, including five suspected cases of acute infection, were categorized in genotype A2, suggesting ongoing active transmission of the virus among the Japanese MSM population. Thus, the genotype A 2 population appeared to be younger, with more acute cases, and infected with an almost genetically identical HBV strain. These two genotypes did not differ significantly in regard other clinical data, such as AST and ALT levels, CD4 ${ }^{+}$T cell count, and HBV and HIV-1 viral loads.

To clarify the detailed epidemiological features of HBV/ HIV-1-coinfected patients, the HIV-1 subtypes and their genetic distances were determined by phylogenetic analyses of three genome regions, gag p17, pol, and env C2V3. All 26 samples were determined as subtype B (Fig. 4A, B, and C), and interestingly, branch patterns and relationships among cases were different from those for HBV. There were six paired cases, demonstrating a significantly close genetic relationship ( $>50 \%$ bootstrap value) in more than two regions. These paired cases were cases 1 and 5, 2 and 15, 6 and 20, 11 and 19, 16 and 25, and 22 and 29, and these connections were not evident in the HBV phylogeny, suggesting different origins of sexual partner between the two pathogens in each pair. An alternative explanation could be that little genetic variation in HBV made it difficult to clarify the genetic relationships between cases. However, there was one discordant pair (cases 1and 5); one case had HBV genotype A, and the other case had HBV genotype C2. Furthermore, the other four HBV genotype C2 cases (cases 9, 17, 20, and 33) were scattered among HIV-1 phylogenies within genotype B HIV-1-infected patients.

HBV strains detected in HIV-1-infected patients from Nagoya are the same viruses found in other parts of Japan. To clarify whether the dominance of genotype A HBV in HIV-1infected MSM is a regional issue in the Nagoya urban area or a more nationwide epidemic, we reconstructed an HBV phylogenetic tree of 26 cases together with HBV sequences col- 


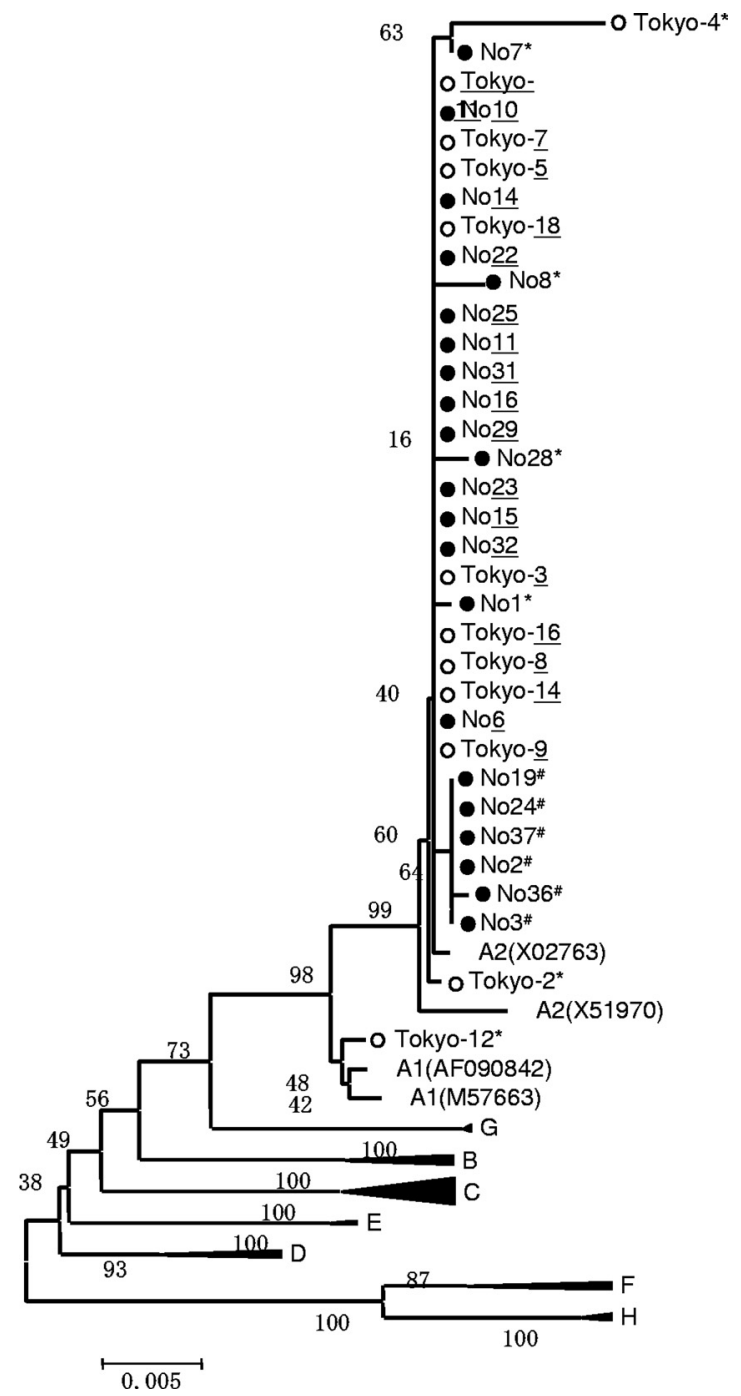

FIG. 5. Phylogenetic tree analysis of $35 \mathrm{HBV}$ region $\mathrm{S}$ sequences, 22 from Nagoya (solid circles) and 13 from Tokyo (open circles). Three genetically different groups are indicated by asterisks, pound signs, and underlining.

lected at a different time and from a different area of Japan, i.e., 12 genotype A sequences from HBV/HIV-1-coinfected patients collected in Tokyo about 10 years before this study (8). As no full genome sequences were available for the Tokyo cases, only the S gene (681 bp [bp 155 to 835]) was analyzed. From the phylogenetic tree pattern, genotype A was classified into three groups (Fig. 5). The first is a group of 21 identical sequences (underlined in Fig. 5). As this group had the largest number of cases and included sequences from both Nagoya and Tokyo, this strain appears to be prevailing nationwide. The second group is a cluster of cases, i.e., cases $2,3,19,24,36$, and 37. As all six cases were from Nagoya, this isolate still seems to be in an endemic status. The third group comprises isolates with longer branches (noted by asterisks), i.e., Tokyo-2, -4 , and -12 and Nagoya- $1,-7,-8$, and -28 . These isolates appear to be quite distinct from the others, suggesting that their origin may not be sexual contact but another route, such as MTCT or transfusions.
The prevailing $\mathrm{HBV}$ genotype $\mathrm{A} 2$ emerged more recently than most other genotypes. To estimate the emergence time of the prevailing genotype A2 strain, we estimated its mutation rate per year and tMRCA. First, the median mutation rate per year was calculated as $3.23 \times 10^{-5}\left(5.62 \times 10^{-8}\right.$ to $9.01 \times$ $\left.10^{-5}\right)$, which is close to those previously reported $(10,18)$. Next, the median tMRCAs of all A strains, A1, A2, and C were determined to be $370.8,88.9,184.3$, and 494.9 years ago, respectively (Table 4; Fig. 6). Thus, the A2 genotype is one of the youngest HBV genotypes.

A lamivudine resistance amino acid HBV mutation detected in an antiretroviral therapy-naïve patient. We clarified not only HBV genotypes but also the incidence of transmitted drug-resistant $\mathrm{HBV}$ among the study patients. Analysis of the amino acid sequence of the HBV RT region showed a combined triple amino acid mutation, rtV173L + rtL180M + rtM204V, which was a mutation causing resistance against lamivudine and its 5-fluoro analogue $\left(2^{\prime}, 3^{\prime}\right.$-dideoxy- $3^{\prime}$-thia-5fluorocytidine), in two patients (patients 5 and 8). However, one patient (patient 5) had been treated with stavudine-lamivudine-efavirenz at the time of sample collection, and thus only one case (case 8) was suspected to be a transmitted HBV drug-resistant case. No HIV-1 drug-resistant virus transmission was detected in the study sample.

\section{DISCUSSION}

This molecular epidemiological study of HBV infection in HIV-1-seropositive patients revealed epidemiological characteristics that were unique compared to those of the general population in Japan. All HBV/HIV-1-coinfected patients were MSM, they had a 10 -fold-higher prevalence $(7.9 \%)$ than that of the general population, and genotype $\mathrm{A}$ was the predominant HBV genotype (31). This distinct HBV epidemic in MSM was first reported in 2001 in other regions of the country $(9$, 36), a decade before our study. Furthermore, phylogenetic analysis of sequences from the two studies, collected in different regions and years, revealed that an identical genotype A strain prevails among the MSM population nationwide.

Considering the status of HBV epidemiology in the general population of Japan, genotypes C and B must have an equal or greater chance to disseminate among the HIV-1-seropositive

TABLE 4. Estimated times of the most recent ancestor (tMRCAs) for HBV genotypes

\begin{tabular}{|c|c|c|c|c|}
\hline \multirow{2}{*}{ Genotype } & \multicolumn{2}{|c|}{$\begin{array}{l}\text { Mean tMRCA } \\
\text { (yr before) }\end{array}$} & \multicolumn{2}{|c|}{$95 \% \mathrm{HPD}^{a}$} \\
\hline & Mean & Median & $\mathrm{L}$ & $\mathrm{H}$ \\
\hline A & $1,294.2$ & 370.8 & 27.1 & $4,046.4$ \\
\hline A1 & 306.6 & 88.9 & 12.4 & 976.4 \\
\hline A 2 & 597.4 & 184.3 & 18.8 & $1,886.2$ \\
\hline B & 345.8 & 88.5 & 4.2 & $1,069.3$ \\
\hline $\mathrm{C}$ & $1,655.3$ & 494.9 & 36.6 & $5,124.7$ \\
\hline $\mathrm{C} 2$ & $1,062.4$ & 308.6 & 20.8 & $3,296.6$ \\
\hline D & 827.2 & 226.6 & 11.2 & $2,469.4$ \\
\hline $\mathrm{E}$ & 163.7 & 38.9 & 4.5 & 539.7 \\
\hline $\mathrm{F}$ & $1,060.8$ & 308.2 & 13.7 & $3,277.4$ \\
\hline $\mathrm{H}$ & 433.8 & 110.1 & 5.6 & $1,303.0$ \\
\hline
\end{tabular}

\footnotetext{
${ }^{a}$ HPD, highest posterior density.
} 


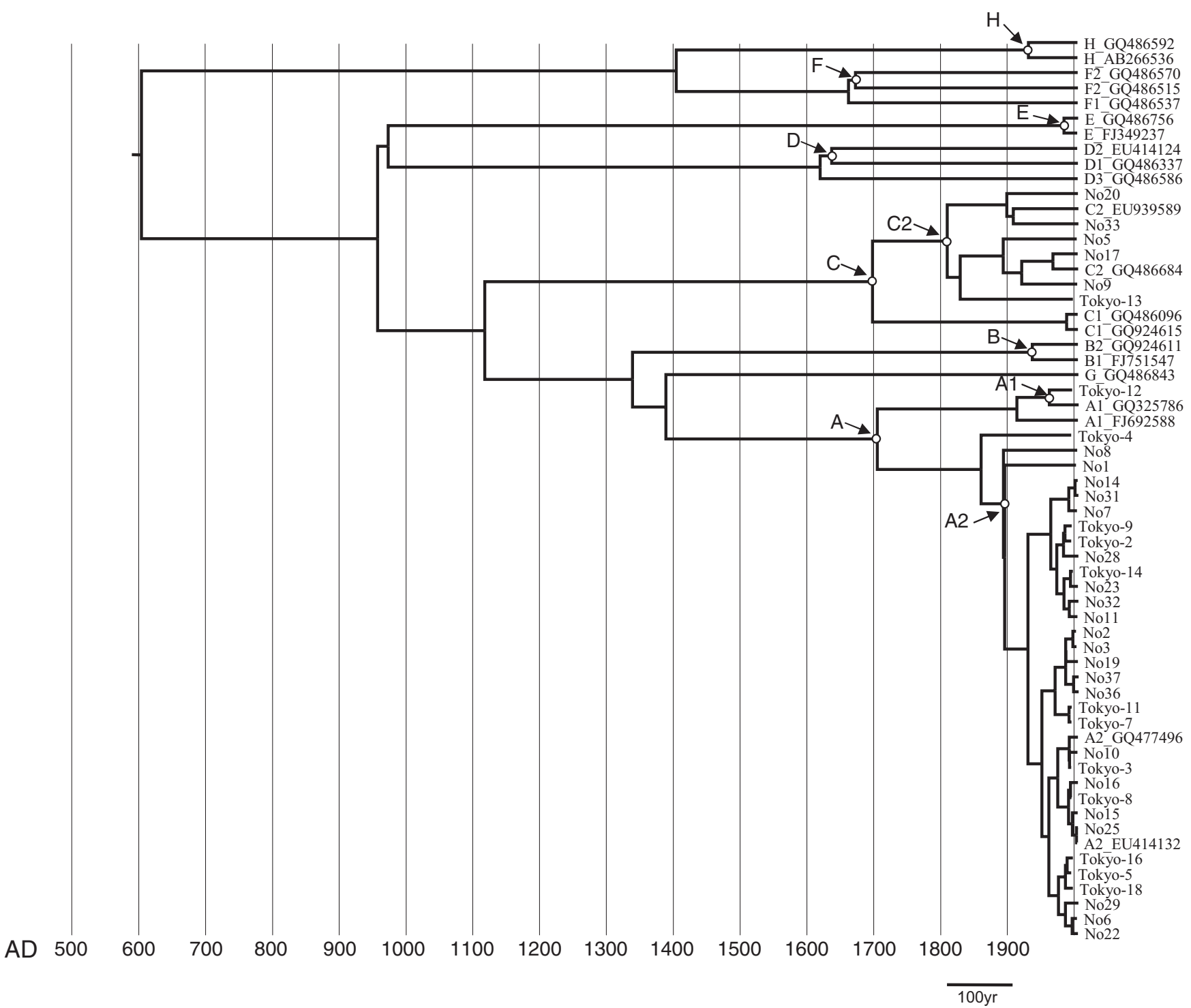

FIG. 6. Maximum clade credibility tree depicted according to median tMRCA. Nodes with open circles are evaluated points for each genotype summarized in Table 2.

MSM population. In fact, we found five genotype C patients in our study sample, and all five patients were MSM. However, because these five genotype $\mathrm{C}$ patients were older and their isolates had longer branches in phylogenetic analysis than the prevailing genotype A isolates, they appear to have been independently infected through either MTCT or blood transfusion events rather than sexual contact. Furthermore, as all five cases were singletons without any genetically close isolates among the samples analyzed, this genotype appeared to be less efficiently transmitted by sexual contact.

Interestingly, predominant genotype A HBV coinfection in HIV-seropositive MSM populations has also been reported in European and South American countries (20, 26, $33)$, suggesting that the prevailing genotype A in HIV-seropositive MSM has become a worldwide HBV epidemic. Regarding HBV genotypes in the HIV-negative population in Japan, genotype A has been increasing, but the major HBV genotype is still $\mathrm{C}$, with genotype A remaining at $3.5 \%$ nationwide and $2.1 \%$ in the Tokai area, which includes Nagoya city (9). Therefore, the prevalence of genotype A $\mathrm{HBV}$ in the MSM population is significantly higher than in the rest of the population.

Thus, it is interesting to discuss the virological advantages disposing this genotype $\mathrm{A}$ isolate to become the major player in HBV/HIV-1 coinfection among MSM. One such advantage might be the higher progression rate (16 to $23 \%$ ) to chronicity of genotype A than of genotype $\mathrm{C}(28,30)$, enhancing its capacity to serve as a source of new infections. As 9 of 26 genotype A-infected patients $(35 \%)$ were $\mathrm{HBcAg}$ IgM positive and 2 had acute hepatitis, it is obvious that genotype A infections are actively ongoing among the MSM population. Though further studies are needed, considering the tMRCA of the prevailing strain A2, the younger age of patients infected with this strain than of those infected with other genotypes, 
and its high prevalence among MSM, this strain may have acquired higher infectivity and efficient transmission through sexual contact.

Another issue we wanted to clarify in this study was the transmission of antiviral drug resistance. We found no antiretroviral resistance in the 26 sequenced cases. On the other hand, we detected two cases with a mutation combination of rtV173L + rtL180M + rtM204V in HBV reverse transcriptase, demonstrating resistance against lamivudine-emtricitabine. One patient was antiretroviral therapy naïve; thus, transmission of drug-resistant HBV is strongly suspected. It is peculiar that the isolate harboring the drug-resistant mutations in HBV was a singleton, considering that genetically identical isolates were prevailing, that there were very low mutation rates that suggest few chances of reverting to wild type, and that there were actively ongoing de novo infections. This finding might be due to resistant viruses being masked by wild-type viruses under untreated conditions, as reported in the case of HIV-1 drug resistance (6). The possibility of minority resistance populations of HBV could be verified by detection with a highly sensitive method.

In conclusion, we clarified the molecular epidemiology of HBV/HIV-1 coinfection in Japan. Our data suggest that ongoing HBV infections lie outside prevention programs targeting the MTCT and blood transfusion infection routes, and they suggest the urgent need for new prevention strategies focusing on the high-risk group of the HIV-1-seropositive MSM population.

\section{ACKNOWLEDGMENTS}

This study was supported by a Research Grant for Research on HIV/AIDS from the Ministry of Health, Labor, and Welfare of Japan (no. H19-AIDS-007, H21-AIDS-005, and H22-AIDS-004).

We thank Yasuhito Tanaka, Nagoya City University Graduate School of Medical Sciences, for helpful discussion and Claire Baldwin for help in preparing the manuscript.

\section{REFERENCES}

1. Allen, M. I., et al. 1998. Identification and characterization of mutations in hepatitis B virus resistant to lamivudine. Lamivudine Clinical Investigation Group. Hepatology 27:1670-1677.

2. Angus, P., et al. 2003. Resistance to adefovir dipivoxil therapy associated with the selection of a novel mutation in the HBV polymerase. Gastroenterology 125:292-297.

3. Drummond, A. J., S. Y. Ho, M. J. Phillips, and A. Rambaut. 2006. Relaxed phylogenetics and dating with confidence. PLoS Biol. 4:e88.

4. Drummond, A. J., and A. Rambaut. 2007. BEAST: Bayesian evolutionary analysis by sampling trees. BMC Evol. Biol. 7:214.

5. Gatanaga, H., et al. 2007. Drug-resistant HIV-1 prevalence in patients newly diagnosed with HIV/AIDS in Japan. Antiviral Res. 75:75-82.

6. Harrigan, P. R., S. Bloor, and B. A. Larder. 1998. Relative replicative fitness of zidovudine-resistant human immunodeficiency virus type 1 isolates in vitro. J. Virol. 72:3773-3778.

7. Johnson, V. A., et al. 2009. Update of the drug resistance mutations in HIV-1: December 2009. Top. HIV Med. 17:138-145.

8. Koibuchi, T., et al. 2001. Predominance of genotype A HBV in an HBVHIV-1 dually positive population compared with an HIV-1-negative counterpart in Japan. J. Med. Virol. 64:435-440.
9. Matsuura, K., et al. 2009. Distribution of hepatitis B virus genotypes among patients with chronic infection in Japan shifting toward an increase of genotype A. J. Clin. Microbiol. 47:1476-1483.

10. Michitaka, K., et al. 2006. Tracing the history of hepatitis B virus genotype D in western Japan. J. Med. Virol. 78:44-52.

11. Miyakawa, Y., and M. Mizokami. 2003. Classifying hepatitis B virus genotypes. Intervirology 46:329-338.

12. Norder, H., et al. 2004. Genetic diversity of hepatitis B virus strains derived worldwide: genotypes, subgenotypes, and $\mathrm{HBsAg}$ subtypes. Intervirology 47:289-309.

13. Noto, H., et al. 2003. Combined passive and active immunoprophylaxis for preventing perinatal transmission of the hepatitis B virus carrier state in Shizuoka, Japan during 1980-1994. J. Gastroenterol. Hepatol. 18:943-949.

14. Nylander, J. 2004. MrModeltest v2. Uppsala University, Uppsala, Sweden.

15. Oda, T. 2000. Further decline of hepatitis B surface antigen (HBsAg) prevalence in Japan. Jpn. J. Cancer Res. 91:361.

16. Okada, K., I. Kamiyama, M. Inomata, M. Imai, and Y. Miyakawa. 1976. e antigen and anti-e in the serum of asymptomatic carrier mothers as indicators of positive and negative transmission of hepatitis B virus to their infants. N. Engl. J. Med. 294:746-749.

17. Orito, E., et al. 2001. Geographic distribution of hepatitis B virus (HBV) genotype in patients with chronic HBV infection in Japan. Hepatology 34: 590-594.

18. Osiowy, C., E. Giles, Y. Tanaka, M. Mizokami, and G. Y. Minuk. 2006 Molecular evolution of hepatitis B virus over 25 years. J. Virol. 80:1030710314

19. Pybus, O. G., A. J. Drummond, T. Nakano, B. H. Robertson, and A. Rambaut. 2003. The epidemiology and iatrogenic transmission of hepatitis $C$ virus in Egypt: a Bayesian coalescent approach. Mol. Biol. Evol. 20:381-387.

20. Quarleri, J., et al. 2007. Hepatitis B virus genotype distribution and its lamivudine-resistant mutants in HIV-coinfected patients with chronic and occult hepatitis B. AIDS Res. Hum. Retroviruses 23:525-531.

21. Rambout, A., and A. J. Drummond. 2007. Tracer v1.4. Institute of Evolutionary Biology, University of Edinburgh, Edinburgh, Scotland. http://tree .bio.ed.ac.uk.

22. Schaefer, S. 2007. Hepatitis B virus taxonomy and hepatitis B virus genotypes. World J. Gastroenterol. 13:14-21.

23. Shafer, R. 2010, posting date. Stanford drug resistance database. http://hivdb .stanford.edu/.

24. Sheldon, J., et al. 2005. Selection of hepatitis B virus polymerase mutations in HIV-coinfected patients treated with tenofovir. Antivir. Ther. 10:727-734.

25. Shiraki, K. 2000. Perinatal transmission of hepatitis B virus and its prevention. J. Gastroenterol. Hepatol. 15(Suppl.):E11-E15.

26. Soriano, V., et al. 2010. Predictors of hepatitis B virus genotype and viraemia in HIV-infected patients with chronic hepatitis B in Europe. J. Antimicrob. Chemother. 65:548-555.

27. Sugauchi, F., et al. 2001. A novel variant genotype C of hepatitis B virus identified in isolates from Australian Aborigines: complete genome sequence and phylogenetic relatedness. J. Gen. Virol. 82:883-892.

28. Suzuki, Y., et al. 2005. Persistence of acute infection with hepatitis B virus genotype A and treatment in Japan. J. Med. Virol. 76:33-39.

29. Swafford, D. 2003. PAUP. Phylogenetic analysis using parsimony (and other methods), version 4. Sinouer Associates, Sunderland, MA.

30. Takeda, Y., et al. 2006. Difference of HBV genotype distribution between acute hepatitis and chronic hepatitis in Japan. Infection 34:201-207.

31. Tanaka, J., et al. 2004. Sex- and age-specific carriers of hepatitis B and C viruses in Japan estimated by the prevalence in the 3,485,648 first-time blood donors during 1995-2000. Intervirology 47:32-40.

32. Tenney, D. J., et al. 2004. Clinical emergence of entecavir-resistant hepatitis $\mathrm{B}$ virus requires additional substitutions in virus already resistant to lamivudine. Antimicrob. Agents Chemother. 48:3498-3507.

33. Trimoulet, P., et al. 2007. Hepatitis B virus genotypes: a retrospective survey in southwestern France, 1999-2004. Gastroenterol. Clin. Biol. 31:1088-1094.

34. Westland, C. E., et al. 2003. Week 48 resistance surveillance in two phase 3 clinical studies of adefovir dipivoxil for chronic hepatitis B. Hepatology 38:96-103.

35. Yang, H., et al. 2002. Resistance surveillance in chronic hepatitis B patients treated with adefovir dipivoxil for up to 60 weeks. Hepatology 36:464-473.

36. Yotsuyanagi, H., et al. 2005. Distinct geographic distributions of hepatitis B virus genotypes in patients with acute infection in Japan. J. Med. Virol. 77:39-46. 